\title{
International Experience of Artificial Intelligence Implementation in the Field of Science and Higher Education
}

\author{
Nikita Aleksandrov* \\ Moscow State Institute of International Relations, University of the Ministry for Foreign Affairs of Russia, Moscow, \\ Russia \\ *Corresponding author. Email: nikitaalex00@gmail.com
}

\begin{abstract}
Modern technological development is characterized by the constant emergence and introduction of innovative technologies in various areas of community, country's economy and governance system development. The obvious benefit of introducing such technologies is the significant transformation and improvement of human activities. Science and higher education are considered to be the principle areas of the government activity and is therefore appealing for change and improvement through modernization. The introduction of innovative technologies improves the sector's overall performance and accelerates its technological development. The article examines the best international practices in introducing artificial intelligence in science and higher education.
\end{abstract}

Keywords: technological development, science, higher education, AI.

\section{INTRODUCTION}

Technological development at the current stage depends largely on the possibility of introducing innovative technologies, as well as their practical application in various spheres. In the context of the digital transformation of all sectors, it is important that the Government closely monitor the introduction of such technologies in order to increase the international competitiveness of industries, improve the well-being of citizens, as well as minimize the risks associated with the introduction of breakthrough technologies [1].

On the background of the COVID-19 pandemic, the strategic importance of artificial intelligence (AI) for governments around the world is becoming more obvious than ever before. From pharmaceutical companies using AI to help develop new medicine and treatments, to the use of AI in education [2]. New technologies are helping countries address the problems caused by the pandemic [3].

The AI is applied in modern context not only to combat pandemics, but also in a wide range of industries. $\mathrm{AI}$ is also playing an increasing role on the international scene in the joint efforts of countries to introduce it for the common good. AI is one of the end-to-end digital technologies used as the crucial tool for the digital transformation of states. The role of AI here also increases, taking into account that the goal of the most common direction of development of the 21 st century (digital transformation) is to increase the welfare of states and ensure sustainable development [4]. For example, the AI4Cities project of the European Union was created to unite the efforts of cities in the application of artificial intelligence to accelerate carbon neutrality.

No doubt artificial intelligence is developing rapidly. This technology has the potential to change our lives by improving health care (for example, better diagnostics to better prevent disease), improving agricultural efficiency, improving safety and providing new opportunities for education [5]. In the last 25 years, the field of AI has achieved success in terms of technological development, theoretical input and impact on education.

The spheres of science and higher education are of fundamental importance as areas for the introduction of AI, because they ensure the formation of both new competencies and knowledge that meet modern needs among citizens, and the general technological development of the country in the sense of developing new technologies for the strategic goals.

In this area, the potential for using $\mathrm{AI}$ is obvious. The most explicit advantages are: 
- ensuring the free and equal use of $\mathrm{AI}$ in education, including eliminating inequalities related to socioeconomic status, gender and ethnicity, geographical location; identifying successful projects or proven effective methods of using AI to overcome the difficulties of access of vulnerable groups to quality education;

- applying AI to improve education and learning improving education management systems, enhanced AI learning management systems and other AI in educational applications, as well as creating new forms of personalized learning that can support teachers and solve problems in the field of education;

- processing of big data in order to analyse it according to set criteria, as well as to predict the development of a certain phenomenon or phenomenon in length and time, based on the retrospective analysis of the data.

\section{MATERIALS AND METHODS}

The object of research is AI technology. The subject of the study is the AI application in public governance of science and higher education under in the context of ubiquitous digital transformation. The purpose of the work is to trace the best international practices as well as the main methods of AI implementation in the field of science and higher education.

To achieve these goals the following tasks are set:

1. Identify leading countries for the development of artificial intelligence in science and higher education;

2. To study the practices and specific cases of AI application in these countries;

3. To summarize the information obtained and identify the leading trends in the development of AI in the field of science and higher education at the present stage.

The methodological basis of the research is theoretical methods, namely, analysis, synthesis, classification, generalization; empirical methods, namely, observation, description.

The study is based on the following sources: scientific articles on the topic, as well as reports from mass media.

\section{RESULTS}

The theoretical study shows that AI technologies have a strong potential for deployment in spheres of science and higher education. Taking into account and adapting the leading countries' experience with AI technology, existing higher education systems which are just getting on the track of digital transformation can be substantially modernized.
With regard to the development of science, the application of AI creates new opportunities for accelerating the process of scientific research, collaborative interaction, research activity and development to a completely new level.

International experience has also shown the need for intergovernmental cooperation in the development and implementation of AI, not only for the sake of interaction in the exchange of experience, creation of completely novel competences and the conduct of joint research, but also in the framework of uniting efforts to combat the global challenges of the 21 st century.

\section{DISCUSSION}

\subsection{AI Application Best Practices}

For a meaningful analysis of the application of artificial intelligence in education, it is necessary to understand worldwide trends in the application of new technologies.

Oxford Insights and the International Centre for Research and Development published an index of the readiness of different countries to use artificial intelligence technologies [6].

The experts conducted the assessment on the basis of various criteria, such as:

- quality of governance;

- innovative strength;

- human capital;

- infrastructure development;

- data availability and representativeness;

- quality of accountability;

- transparency, etc.

The United States of America ranks first in the index published in 2020. Most of the remaining places in the top ten are occupied by European countries, which reflects the fact that North America and Europe are the regions with the highest indicators in general.

The US is known for its innovations in the private sector, and "Silicon Valley" is almost synonymous with advanced technologies. Meanwhile, although Europe does not yet have technology centers on a par with the United States, there is a high concentration of national AI strategies in Western Europe, supported by the European Union's regional strategy outlined in its white paper "Artificial Intelligence - a European Approach to Excellence and Trust" [7], published in 2020.

Thus, the European Union supports a regulatory and investment-oriented approach to promote the 
introduction of $\mathrm{AI}$ and eliminate the risks associated with certain types of use of this new technology [8].

In general, artificial intelligence can be described as a set of technologies that combine data, algorithms and computing power [9]. That's why advances in computing and the increasing availability of data are key factors in modern development.

Europe seeks to combine its technological and industrial advantages with a high-quality digital infrastructure and a legal and regulatory framework based on its fundamental values. All this is done to become a global leader in the field of economics and innovation.

China, which has the ambition to challenge the US for global leadership in artificial intelligence, ranks surprisingly low in index $19^{\text {th }}$. Russia is ranked $33^{\text {rd }}$.

In the sphere of higher education, innovations in digital education will help to make better use of data and technologies based on AI. These include training and forecasting analysts. The main goal of Governments is to improve education and learning systems and to bring them into line with the digital age. It is also important to raise awareness of $\mathrm{AI}$ at all levels of education in order to prepare citizens to make informed decisions that will be increasingly influenced by AI.

More specifically, among foreign AI practices, the most interesting and sought after are [9]:

- Smart assistants (Great Britain), as a tool that presents options for solving student requests according to a typical scenario;

- Recommendations for the selection of enrollee (USA) [10]. This is a prediction of the areas of scientific interests and academic performance based on the student's portfolio;

- Recommendations on measures to support researchers (Germany), forming proposals for sending applications for the provision of support measures based on their scientific interests;

- Chatbots (Rep. Korea). They can be described as an "entry point" to the "electronic dean's office", through which students can send a request concerning their daily necessities;

- Proactive interaction (Netherlands), which is the prevention of difficulties that may arise in the learning process and the development of mechanisms to overcome them.

Proctoring (Singapore). This is a system for monitoring and verifying students in the case of final assessment and grading takes place in an online format.

\subsection{Essence of Digital Transformation in Science and Higher Education}

It is important to note that the introduction of $\mathrm{AI}$ should take place solely under the umbrella of a single goal - the digital transformation of the industry. It is therefore necessary to delve more deeply into the essence of digital transformation of the science and higher education sphere.

The conceptual direction of digital transformation defines the digital unity of education and science for the transition to data-driven governance. Fundamentally, digital transformation of the sphere involves education with the help of digital search and research tools. Digital transformation can become a tool for improving the quality of education, since it will be possible to introduce a practice-oriented approach to teaching everywhere [11].

For countries starting to implement AI, it is possible to consider the transition to predictive and prescriptive analytics as a tool for preventive governance. This will allow the country to move out of the catching-up position, into the position of a leader who sets trends. It will also transform the governance process and significantly improve the quality of decision-making [12]. It is also important to consider the digital collaboration education-science-business, whose goals are aimed at achieving the well-being of society. It is supposed to combine the efforts of the real sector of the economy, science and education, since these segments should not be disconnected from each other.

Training of human resources, who will have to work with innovative technologies (AI) and change traditional behavior and routines, also plays an important role in such processes. The ubiquitous introduction of innovative technology inevitably brings about numerous and varied changes in the economic, political and social spheres of society. New challenges require the training of professionals to meet the increased technological requirements. According to statistics, the share of employees whose functions are directly related to the development and application of digital technologies and tools in the US is $3.8 \%$, and in the EU $-3.7 \%$. In this regard, the need to train and retrain personnel to meet the needs of a new technological environment is becoming more urgent than ever before.

According to a survey of 5,600 managers of global companies on the topic of skills development conducted by IBM, half of the respondents believe that the companies themselves are responsible for building the necessary skills in their employees, and only $39 \%$ believe that staff members themselves should develop and maintain their professional skills [13]. Therefore, major international companies are actively introducing new educational programs and courses to improve the skills of their employees. Thus, Google implements a number 
of free online digital skills training programs and activities. Since 2015, the company has been implementing the program «Grow with Google» on digital skills training, which is implemented through online and offline channels in the U.S. and $28 \mathrm{EU}$ countries and other states. The Digital Workshop, an interactive learning platform, provides employees with a plan specifically designed to fill gaps in their knowledge and at the desired learning rate. The company also develops relevant courses and training programmes in IT and data analysis. For example, the Machine Learning Crash Course (MLCC) or the Coursera program «Google IT Support Professional Certificate» will provide basic IT knowledge and skills.

\subsection{AI Development Global Trends}

Based on the practical and theoretical experience cited above [14], the key trends in the development of AI in the science and higher education sector were formulated:

- Individualization and personalization. As a rule, it incorporates a selection of courses in accordance with the scientific interests of the learner [15]. It is also important to adapt teaching methods to the needs of the student. Special emphasis is placed on the development of new disciplines based on retrospective data analysis using AI technologies;

- Platform approach. This trend involves the development of digital platforms for online courses for students and the development of course recommendations in line with scientific interests. In order to achieve this approach, it is crucial to establish common platforms for research collaborations with functional recommendations for collaborative research, as well as available researchers' support measures;

- Formation of unified information ecosystem between educational and scientific organizations [16]. This implies creating scientific connectivity through the use of advanced information technologies (AI), as well as integrating the necessary aspects into a single digital portfolio in educational and research activities. The socalled «digital trail» of learners and researchers will allow to update the most important subject areas and, if necessary, to continue and develop existing research, achieving results at a higher speed;

- Digital profiles. Here the main focus concentrates on the development of a common profile (both research and academic/educational) with the following functions: search for researchers/scholars to perform R\&D, search for collaborators; online research infrastructure/equipment booking (in some cases, for remote study conduction), unified register of research grants and support measures.
- Virtual research assistants [17]. This tool will automate and speed up «routine tasks», analyse big data and allow smart selection of materials on scientific topics, organized support measures and ways to obtain them effectively.

All the above-mentioned trends of AI development in the context of digital transformation provide the main functional areas of application of AI in science and higher education. They not only define a common agenda in modern development, but can also be used as landmarks or "target states" of AI development.

\section{CONCLUSION}

The study showed that the development of AI is proceeding fairly rapidly in the field of science and higher education, as one of the main branches of development of modern countries. The potential and possibilities for AI applications are already being found in various countries. However, the AI development and implementation process is still under way, which means that there is considerable potential for the development of such technologies to become more complex, deeper and more relevant.

The objectives of the study are considered to have been achieved. First, the leading countries on the level of AI readiness and implementation in the sphere of science and higher education were identified along with practical cases that have already been applied and are yielding results. Next, on the background of theoretical pillars of digital transformation the leading trends of AI application were identified and summarized.

In the end, AI technologies have significant potential for deployment in science and higher education, and there are already successful examples of such technologies in international experience. The main directions of AI technology implementation are creation of a single digital profile, support of research activities.

The results of the research generally show that the introduction of AI technologies into science and higher education sphere creates a certain value, in particular, it helps to strengthen and accelerate the economic and social development of the country.

\section{REFERENCES}

[1] S. Kamolov, I. Molchanovskaya, E. Kaunov, Artificial intelligence as a strategic instrument of economic development of Russia and improvement of its public administration, In: E3S Web of Conferences. EDP Sciences, 2021, p. 04002.

[2] P. V. Chetyrbok, Artificial intelligence in remote education. Remote education technology, $2018 \mathrm{pp}$. 91-95.

[3] S. G. Vasin, Artificial intelligence in public governance. Management Journal 3(17) (2017). 
[4] S.G. Kamolov, A.A. Smagina, Digital Innovation Technologies in Public Governance: Budget Geolocation System. Lecture Notes in Networks and Systemsthis link is disabled 1292020 pp. 467-475.

[5] S.G. Kamolov, Digital public administration: trends and risks. Digital public governance: trends and risks, In: S.G. Kamolov (Ed.), Journal of Constitutional History 33(1) 2017 pp. 185-194.

[6] O. Insights, Government Artificial Intelligence Readiness Index 2020. https://static1.squarespace. com/static/58b2e92c1e5b6c828058484e/t/5f7747f2 9ca3c20ecb598f7c/1601653137399/AI+Readiness +Report.pdf.

[7] European Commission. On artificial intelligence-A European approach to excellence and trust 2020.

[8] G. I. Kolesnikova, Artificial intelligence: problems and prospects. Videonauka 2(10) (2018).

[9] B. Goertzel, Artificial general intelligence: concept, state of the art, and future prospects. Journal of Artificial General Intelligence 5(1) (2014) p. 1.

[10] S. Vincent-Lancrin, R. Van der Vlies, Trustworthy artificial intelligence (AI) in education: Promises and challenges 2020 .

[11] S. Custer, et al. Toward Data-Driven Education Systems: Insights into Using Information to Measure Results and Manage Change. Center for Universal Education at The Brookings Institution 2018.

[12] S.G. Kamolov, P.V. Artemova, V.E. Berezko, Shaping the systems of digital public governance of the digital economy in Russia and abroad. Studies in Computational Intelligencethis link is disabled 826 (2019) pp. 435-442.

[13] A. Ferrari, Digital competence in practice: An analysis of frameworks. JRC IPTS 2012. DOI: https://doi.org/10.2791/82116.

[14] J. Borenstein, A. Howard, Emerging challenges in $\mathrm{AI}$ and the need for AI ethics education. AI and Ethics 1(1) (2021) pp. 61-65.

[15] H. B. Santoso, P. O. H. Putra, S F. F. F. Hendra, Development \& Evaluation of E-Learning Module Based on Visual and Global Preferences Using a User-Centered Design Approach. International Journal of Emerging Technologies in Learning 16(15) (2021).

[16] V. Terziev, V. Klimuk, Modelling the forms of international scientific and educational cooperation 2021.

[17] Y. Zhong, X. Liu, Design and Research of Virtual Reality Teaching Assistant System for Colleges and
Universities Experimental Course Under the Background of Wisdom Education, In: 2021 International Conference on Intelligent Transportation, Big Data \& Smart City (ICITBS). IEEE, 2021, pp. 533-35. 